\title{
Intracardiac Mass Detection and Classification Using Double Convolutional Neural Network Classifier
}

\section{DOI:10.36909/jer.12237}

\author{
${ }^{* 1}$ A.Manikandan and ${ }^{2}$ M.Ponni Bala \\ ${ }^{1}$ Assistant Professor, Department of ECE, Vivekanandha College of Technology for Women, \\ Tiruchengode, India \\ ${ }^{2}$ Associate Professor, Department of EIE, Kongu Engineering College, Erode, India \\ ${ }^{1}$ mani85a@gmail.com, ${ }^{2}$ myphdpaper@outlook.com \\ Corresponding Author\& mail id: A.Manikandan and mani85a@gmail.com
}

\begin{abstract}
Intracardiac masses identification in the images of echocardiogram images in one of the most essential tasks in making the diagnosis of cardiac disease. For making the improvement in accuracy over the diagnosis as a new complete method of classifying the echocardiogram images automatically which is based on robust back propagation neural network algorithm in being proposed for distinguishing intracardiac thrombi and tumor. Initially, the cropping over the specific region is done in order to make the definition of the mass area. Later on, as the second step the processing of globally unique denoising technique is being implied for the removal of speckle and in order to make the preservation of anatomical structured component in the image. This is defined in terms of preprocessing and it is carried out by Patch-based sparse representation. Subsequently the description of the mass contour and its interconnected wall of the artery are being done by the segmentation mechanism denoted as Linear Iterative Vessel Segmentation model. As the prefinal stage, the processing of boundary, texture and the motion features are being carried out through the processing by double convolutional neural network (DCNN) classifier in order to determine the classification of two different masses. Totally 108 cardiac masses images are being collected for accessing the effectiveness of the classifier. It is also realized with the various state of the art classifiers as projected the demonstration of the greatest performance that has been disclosed with an achievement of $98.98 \%$ of accuracy, $98.89 \%$ of sensitivity and $99.16 \%$ of specificity that has been resulted for DCNN classifier. It determines the explication that the proposed method is capable of performing the classification of intracardiac thrombi and tumors in the echocardiography and ensures for potentially assisting the medical doctors who are in the clinical practice.
\end{abstract}

Keywords: Cardiovascular Sickness, Echocardiogram, Cardiac Masses, Patch-based sparse representation, Linear Iterative Vessel Segmentation, Multiscale Local Binary Pattern, Robust Back Propagation Neural Network.

\section{Introduction}

Intracardiac masses are irregular structures found in or near the heart. This structure can cause real cardiovascular problems and requires careful discovery and short-term resection and 
treatment. There are two basic types of dominant intracardiac masses: tumors and blood clots. Cancer shows the growth of parts of the body, Jomilham, through unpredictable tissue improvement [1-2]. Even though intra-cardiac differ only in pathology, they function similarly in electrophysiology. The Echocardiogram intracardiac tumor (Figure 1(a)) and thrombi (Figure 1(b)) are shown in Figure 1.

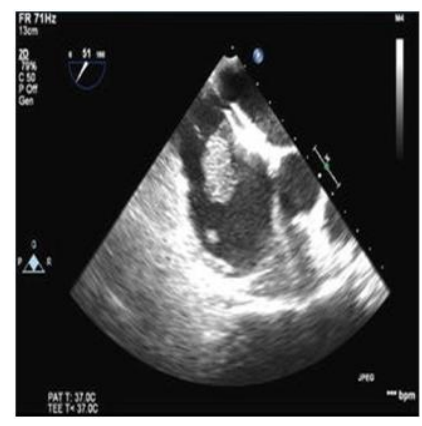

a) Intracardiac Tumor

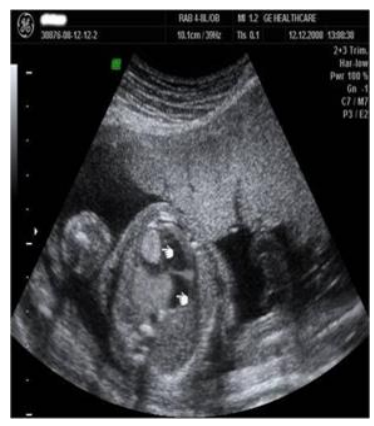

b) Intracardiac Thrombi

Figure1:(a) Intracardiactumor and (b) intracardiac thrombi

In numerous hospitals or medical clinics, echocardiograms recognizing bits of confirmation are finished by cardiologists manually. These types of diagnosis are very time consuming. Approval depends on the quality and skill of the image, as well as the experience of the cardiologist. Therefore, the requirement for a computerized recognition is expanding, which can improve analytic precision and guide in which a specialist should be recommended [3-5]. Ultrasound image testing has been applied to the computer-based end of cardiovascular disease, for example, uncovering important ultrasound highlights in early stroke forecast, structuring rule-based choice emotionally supportive networks in the finding of the coronary vein, and appling square coordination systems to intra-cardiac masses [5].

Notwithstanding, it is a difficult region for intra-cardiac mass detection. Because the relative echocardiogram inception of the two masses and the picture quality are hazardous, including the vast measure of speckle noise, the sign forgets about the rarities and missing shapes [6]. So, a novel system is required to describe the intra heart tumor and thrombi in echocardiograms.

\section{Research Background}

This section discusses the literature survey based on conventional intracardiac mass identification and classification methods.

Cardiovascular tumors are irregular patterns of the coronary heart. There are a few kinds of coronary tumors. The assessment of ultrasound images has been utilized effectively in the computer-aided design of cardiovascular infections, to distinguish the proof of necessary ultrasound emphasis in the early forecast of stroke [7], in the construction of an aid system with the selection based on fuzzy rules for the prognosis of coronary artery disease [8], and in the use of adaptive blocking adaptive methodologies in the dynamics of the wall and the plate of the 
carotid artery [9]. However, it is always difficult to differentiate the heart masses from comparable echocardiogram images from a defective image due to the presence of different weights and a high degree of noise, character drop, distortion, and lack of contouring. The neuronal culture was recommended by AnirbanMukhopadhya to identify and phase out echocardiograms of intracardiac tumors by the hundreds [10].

A fully automated classification technique has not been suggested in the past near the distinction of intracardiac loads on echocardiograms. This classification consists of four stages, such as noise removal, segmentation, feature extraction, and mass classification. Usually, different types of noises are present in ultrasound images, the structure of which also contains beneficial anatomical information. In addition to extracting the essential facets of the image and protecting the anatomical details, it is necessary to obtain a pleasant diagnosis. Usually, different types of noise removal strategies are available, such as pixel intensity correction $[11,12,13]$, median filter-based preprocessing [14], speckle-decreasing anisotropic diffusion (SRAD) found preprocessing [15-16], and preprocessing based on wavelet transformation strategies[17]. As nearby noise decreases procedures, they balance the standard inhomogeneous zones and keep up the edges and realities. These filters are effective at removing stains, but they also remove great small runs. Regarding segmentation, the massive contour artifacts reduction [18], the staging [19], the Animated-Looking Mannequin [20], the fuzzy techniques [21], and the sketch reduction [22] were evaluated. Despite the way that these strategies may be valuable for express different kinds of images, they fail in partitioning heart masses and the improvement of the heart chamber at a particular stage in the cardiovascular cycle.

At the systolic level, the chamber limits so much that it loads up with an intracardiac mass, covering the atrial divider as far as possible. Exciting endeavors have been made in computer-aided classification applications, such as the synthetic multilayer ANN [23-24] and SVM [25]. In [26], a scattered illustration approach is implemented for the classification of tumors and thrombi in intracardiac ultrasound images.

In [27], F-FDG intake in cardiac tumors can distinguish benign and malignant cardiac tumors and predict survival. In [28-29], discussed the latest developments in the fields of cardiovascular imaging and related machine learning in the fields of video acquisition and reconstruction, image analysis, diagnosis, evaluation, and prognostic information derivation. In [30], the prevalence of malignant diseases, which are increasing globally. The development of modern diagnostic tools guarantees improved early detection.

\section{Proposed Intracardiac Masses Detection and Classification}

This section discusses the operation of the proposed intracardiac mass detection and classification system based on the Patch-based sparse representation, GLCM-LBP based DCNN classifier. The proposed system consists of five stages, such as frame separation, noise removal, segmentation, feature extraction, and mass classification. To deal with the intracardiac issue the proposed work thought of the course of action of a DCNN Classifier for intracardiac disease 
identification and classification. This technique is needed to manage the differentiation of patterns. The structure first transfers all images to a specific level, and transfer all images to a specific level. Examples of noise-free usage using Patch-based rare representations are much clearer, with different types of features. The extracted features could be used for making the classification model. This classification model framework, we can finally predict the intracardiac infection masses. Finally, the proposed framework recommends clinical treatment or guidance dependent on our anticipated intracardiac disease result. In this research work, a total of 340 images were tested. In this process, 240 images were collected from Vinayaka Mission's Kirupananda Variyar Medical College \& Hospital, Salem, Tamilnadu.

\subsection{Frame Separation}

The Echocardiogram is one of the major presentations of mass acceptable proof and classification into the masses of the heart, which is the number of frames that take the form of shots, and the multitude of shots convey the scenes, including mixing different scenes to make the video format. To examine any video, first, we need to focus on the characteristics of the frames and then dismember the properties of the video. The video does this to frame the alteration procedure. The working flow chart of frame conversion is shown in Figure 2. 


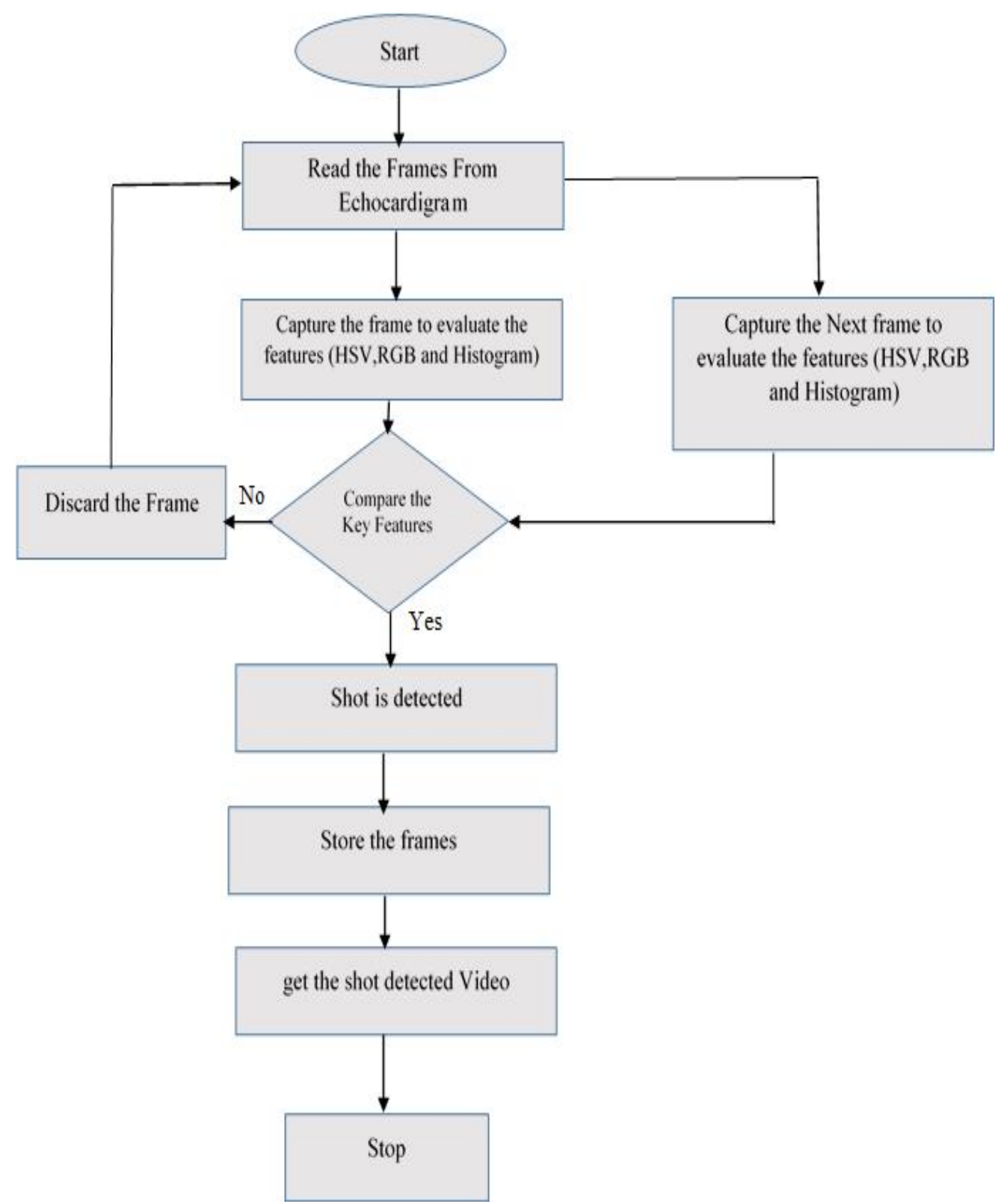

Figure 2: Flow Chart of Frame Separation

\subsubsection{Frame Decomposition}

Cardiologists obtain core ultrasound movements when diagnosing a pre-considered marginal mass. The average length of cardiac ultrasound classification is about 3-4 seconds. The frame rate is 39 frames per second. Each frame has $480 \times 640$ pixels. Contrasted with the movement of the heart in two dynamics, these texts and symbols stand out. After consideration, the two dynamic photo frames have static data which is, empty while the area is checked. This is an area that has the remains of the heart movement. With it, a brief description of the free zone is considered as part of the analysis, and a square shape covering the plot is recognized. At long last, the first image is trimmed to save the filtered locale for additional investigation. 


\section{Patch-based sparse representation}

The nearness of speckle noise in echocardiogram images disrespects the image quality and influences the edges and exquisite details of the image, which makes demonstration progressively troublesome. In this manner, image pre-processing is fundamental for the removal of speckle-noise, which helps in the improvement of computer-aided classification of intracardiac diseases. Therefore, Patch-based sparse representation has been utilized in image pre-processing for this work. First, an adaptive median filter is used to detect the heart image and obtain the initial guidance image. Second, you will learn rare patch-based sparse representation from the images in this guide; third, a normalization method with weighted $l_{1},-l_{1}$ is provided to punish the candidate noise that is heavier than the remaining pixels. The alternating direction minimization algorithm is derived to solve the normalized model. Experiments were carried out under speckle noise levels of $30 \%$ to $90 \%$. The simulation results show that the proposed method has overall changes and wavelets in maintaining the structural similarity with the edges of noisefree images, and has better performance. The existing adaptive vector median filter used in core ultrasound images is also used to reduce image noise. It is a nonlinear filter described in the spatial domain, as shown in Figure 3.

In the experiment, we found two reasons for the degraded noise reduction performance. First of all, many image structures are lost in the guided image, which affects the learning of appropriate similarity. Second, since the original PANO-based $l_{1},-l_{1}$ normalized model did not distinguish between noise and noise-free pixels. 


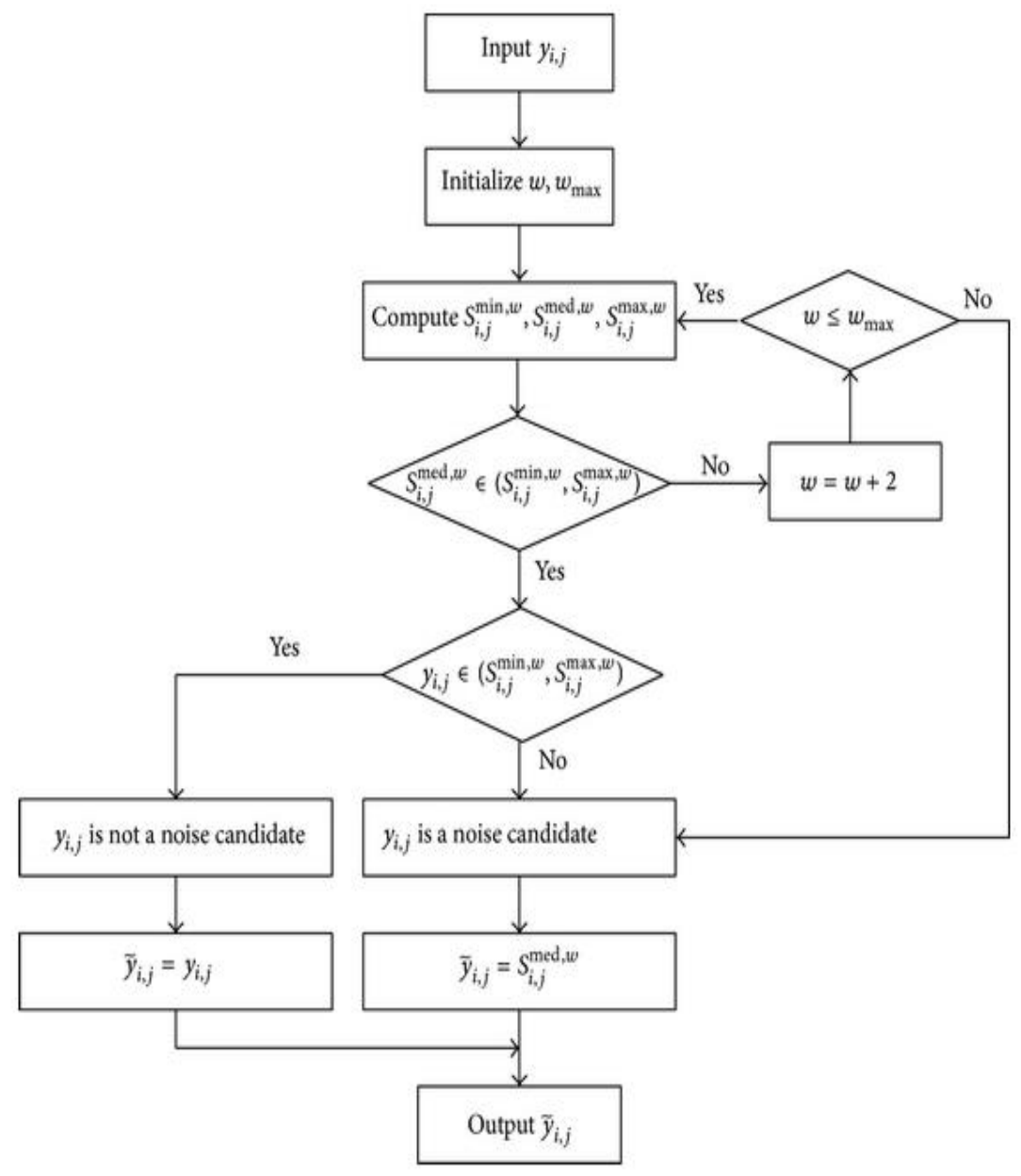

Figure 3: Flow chart of Patch-based sparse representation

Based on the discussions, the preprocessed echocardiogram images are depicted in Figure 4.

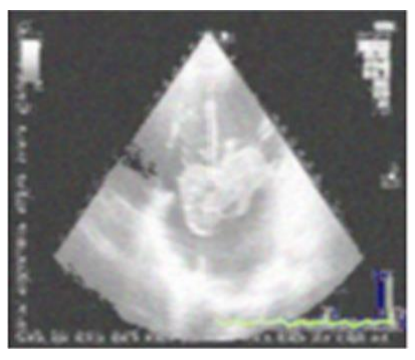

(a) Input cardiac Image

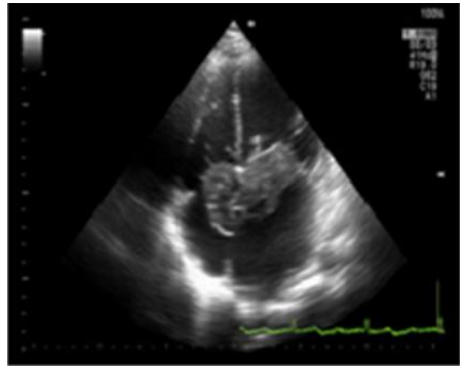

(b) Preprocessed Image

Figure 4: Sampled Result of Patch-based sparse representation

The input (4a) and preprocessed result (4b) images are shown in Figure 4. By utilizing the Patch-based sparse representation, the preprocessed image looks like a more energetic view as compared with the input image. Further, the preprocessed image is used for segmentation. The working procedure of segmentation is discussed in the accompanying section. 


\subsection{LINEAR ITERATIVE VESSEL SEGMENTATION}

First, interpret the image using the neutrosophic set, which calculates the spatial information of the image. Then, the error in the image defined by combining the spatial information and the intensity information also uses this value to configure the error filter. Indeterminacy filters reduce the uncertainty of their respective strength and spatial information. A graph of the image, use the uncertain filtered value to represent the weight of each pixel, and use the neuron value to redefine the energy function. The algorithm is used to obtain the final segmentation result.

$\delta_{i}=\left\{\begin{array}{c}\min _{j}\left(d_{i j}\right) \text { if } \rho_{j}>\rho_{i} \\ \max _{j}\left(d_{i j}\right) \text { if } \rho_{i} \text { is the highest density }\end{array}\right.$

Where $\rho_{i}, \rho_{j}$ represents the density of point $i$, and $j$ and $\delta_{i}$ is the distance.

The result of segmentation in this work is produced using an ultrasound intracardiac image with three classes. Each type contains a three-dimensional component vector related to every pixel alongside the class name as shown in Figure 5.

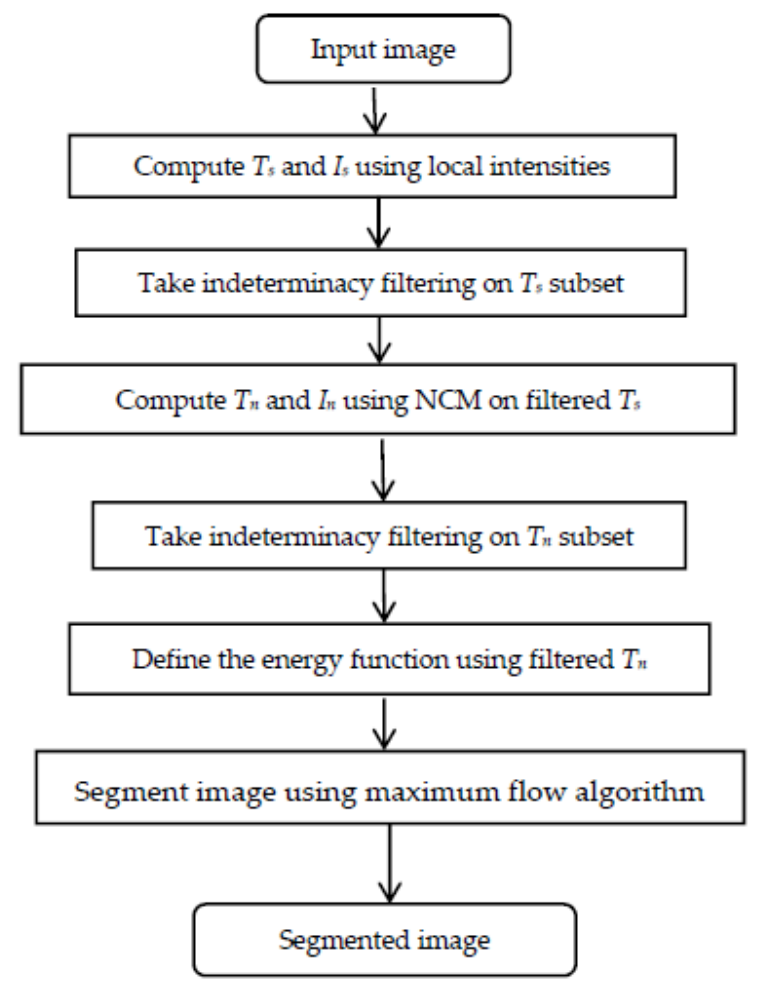

Figure 5: Flow chart of Linear Iterative Vessel Segmentation algorithm 


\subsection{Feature Extraction: Multiscale Local Binary Pattern Strategy (MS-LBP) with GLCM}

This paper proposes a content-based image retrieval method, which focuses on the extraction and reduction of multiple features. Discrete wavelet transform is first applied to RGB channels to extract the approximate values and precise coefficients of the image multi-level decomposition. Therefore, both the approximation and the exact coefficient are computationally valid, and are suitable for the main rotating local binary mode that presents a rotation-invariant texture. For patches in the local neighborhood, measure the descriptor as a reference and obtain the image of the rotation invariant function. The proposed method contains the complete structure information extracted from the local binary pattern, and uses the size information to extract additional information to obtain additional discriminative power. Then use GLCM technology to capture the dominant rotated local binary mode image and extract the statistical characteristics for texture image classification.

To develop feature indicators, the MS-LBP approach first involves a asymmetrical neighborhood circularly around each pixel and a dark estimate of the neighboring pixels in the focal pixels' dim estimation. For example, the threshold yield has two types of data: character and scale. Be that as it may, the scale data, gives the size of the difference, for example, the amount is higher than the neighboring pixel or smaller than the confidence inside the pixel. To achieve dark surface invariance, the LBP approach uses only local neighborhood comparisons for binarization. Therefore, a binary property vector is given by dividing the amount by one of the neighboring pixels is more interesting than the internal pixel. In any case, the value of 0 is allocated. Mathematically, the MS-LBP feature vector is descriptive as mentioned in equation (2).

$$
V=v\left(s\left(g_{o}-g_{c}\right), s\left(g_{1}-g_{c}\right) \ldots \ldots \ldots \ldots \ldots s\left(g_{p-1}-g_{c}\right)\right) \ldots \text { (2) }
$$

Where $g_{c}$ tells the estimation of the dimming of the average pixel and $g n,(n=0, \ldots P-1)$ demonstrates the darkening of the neighboring pixel inside the radius $\mathrm{R} ; \mathrm{P}$ is the complete number of neighbors in the nearby picture adjustment. The binary feature vector is the iterative binomial factor and afterward finishes up the code that depicts the spatial structure on the neighborhood picture that has changed in the equation (3) and (4) respectively.

$$
\begin{gathered}
M L B P_{P, R}=\sum_{n=0}^{P-1} S\left(g_{n}-g_{c}\right) 2^{n} \ldots \\
s(x)=\left\{\begin{array}{l}
1 \quad x \geq 0 \\
0 x<0
\end{array} \ldots(4)\right.
\end{gathered}
$$

Where $\mathrm{s}(\mathrm{x})=$ Local neighboring pixel differences, i.e. $\mathrm{g}_{\mathrm{n}}-\mathrm{g}_{\mathrm{c}}=0$ (when its argument is negative, otherwise one). The algorithm steps of MS-LBP are discussed in below.

The local binary patterns, called histograms in the current work, extract features and do not provide pattern frequencies. Use dominant rotation local binary mode (DRLBP), we use GLCM in all directions and lengths, and introduce improved feature extraction methods. The first DRLBP is calculated using discrete wavelet transform, with multi-scale resolution accuracy and approximation factors obtained from the red, green, and blue components of the original 
image, as shown in Figure 6. Then, considering the four directions and two distances, the GLCM pattern diagram is obtained. Explore different combinations obtained by correlating different distances and directions to obtain better extraction capabilities.

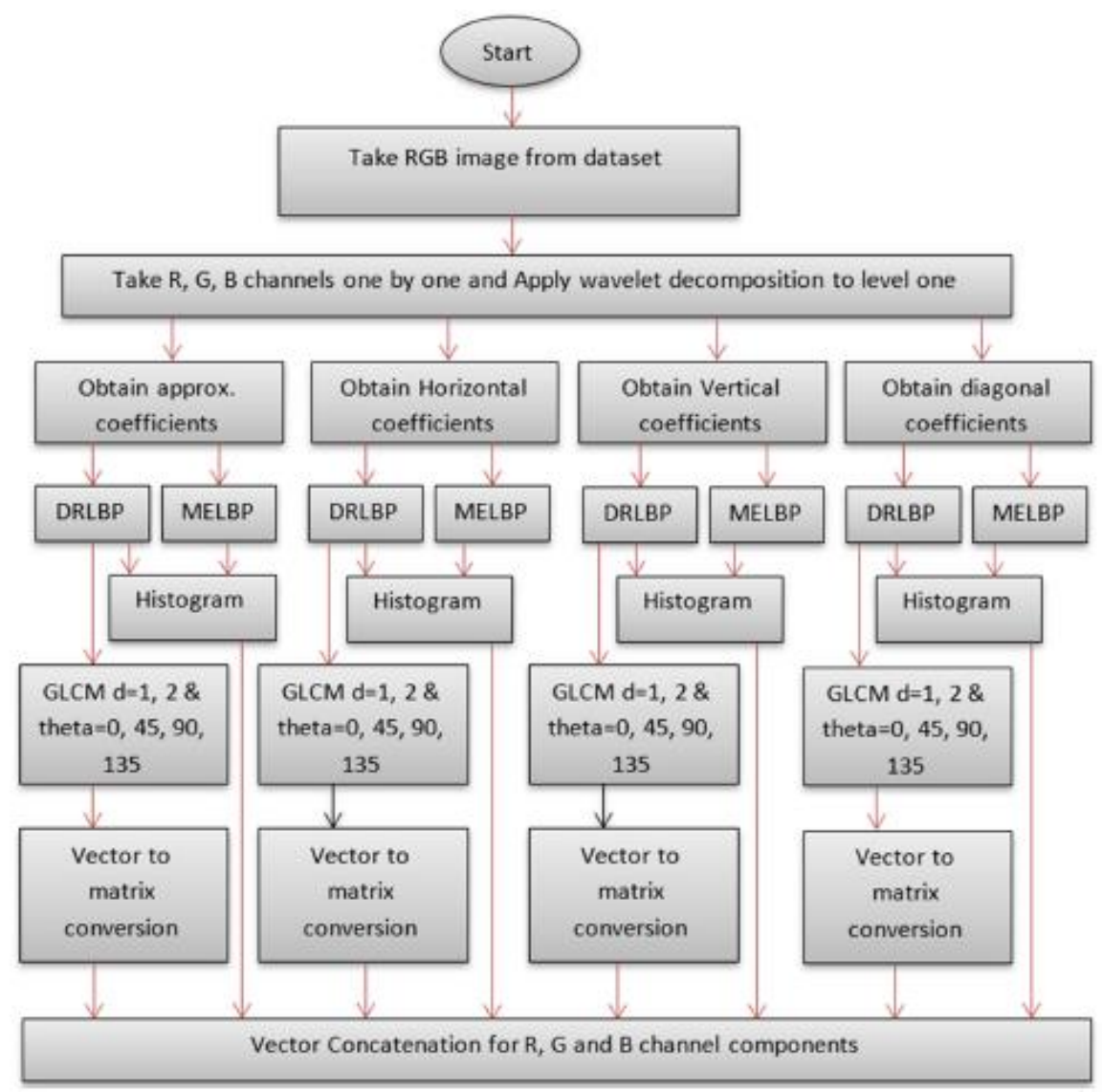

Figure 6: Flowchart of feature extraction phase

The feature extraction algorithm computes the Selected Features similarity at each pixel and finally calculates the similarity of the cumulative pixels. The proposed method extracts 12 features, such as PSNR, MSE, contrast, variance, homogeneity, collection, skewness, energy, mean, entropy, standard deviation, and kurtosis. The results of feature extraction for various images are depicted in Table 1. 
Table 1: Result of Feature Extraction with various sample images

\begin{tabular}{lccc}
\hline \multicolumn{1}{c}{ Features } & $\begin{array}{c}\text { Normal } \\
(\text { Mean } \pm \text { SD })\end{array}$ & $\begin{array}{c}\text { Cardiac Tumor } \\
(\text { Mean } \pm \text { SD) }\end{array}$ & $\begin{array}{c}\text { Cardiac Thrombi } \\
(\text { Mean } \pm \text { SD) }\end{array}$ \\
\hline Standard deviation & $1.5643 \pm 0.4011$ & $0.0818 \pm 1.9196$ & $0.3145 \pm 0.0218$ \\
Contrast & $0.0854 \pm 0.0142$ & $0.02834 \pm 0.0324$ & $0.0742 \pm 0.0672$ \\
Correlation & $0.7298 \pm 0.0126$ & $0.1266 \pm 0.2407$ & $0.5603 \pm 0.0236$ \\
\hline Energy & $1.7843 \pm 0.0248$ & $0.7856 \pm 0.3889$ & $0.9362 \pm 0.0278$ \\
Entropy & $2.2432 \pm 0.1737$ & $0.7250 \pm 0.9234$ & $0.5213 \pm 0.1340$ \\
\hline Homogeneity & $2.1247 \pm 0.0207$ & $0.9367 \pm 0.0158$ & $0.9349 \pm 0.0054$ \\
LBP1 & $3.2234 \pm 0.1361$ & $1.4278 \pm 0.4938$ & $1.7799 \pm 0.217$ \\
LBP2 & $3.3437 \pm 0.0252$ & $1.5358 \pm 0.0340$ & $1.7743 \pm 0.894$ \\
LBP5 & $1.7631 \pm 0.1972$ & $0.4963 \pm 0.6536$ & $0.6498 \pm 0.3908$ \\
\hline LBP9 & $1.9570 \pm 0.0324$ & $0.4676 \pm 0.0504$ & $0.5564 \pm 0.1113$ \\
\hline Mean intensity & $3.1278 \pm 0.02719$ & $0.9413 \pm 0.0218$ & $1.26134 \pm 0.0267$ \\
\hline
\end{tabular}

\subsection{Double Convolutional Neural Network (DCNN)}

The main purpose of the proposed Double Convolutional Neural Network (DCNN) is to provide a model similar to the internal system of cardiac quality classification, In order to analyze various systems based on experience, the ultimate goal of these algorithms is to be able to generate the possibility of learning streams in artificial networks, and provide deep learning to diagnose the network like the human brain. The network contains some new and unusual features that can improve performance and reduce training time. High network size has become an important issue, and several effective techniques have been used to prevent overfitting. The final network consists of 5 layers and 3 layers, and depending on the depth, removing each convolutional layer (each containing more than $1 \%$ of the model parameters) will result in a lower yield. The net has eight layers of weight. The first five are convolutional, and the remaining three are fully connected.

After the explanation, the model can explain the Double CNN architecture. As shown in the figure below, there are eight layers with weights in the model, the first five layers are the same Convolution layer, and the remaining three layers are fully connected layers. The second, fourth, and fifth convolutional layer kernels are connected to the previous MAP kernel on the same GPU. The third kernel in the convolutional layer is connected to all kernel maps in the second layer. Neurons are connected from neurons in the fully connected layer to previously connected neurons. The response normalization layer follows the first and second convolutional layers. The largest pooling layer follows the full layer and the fifth convolutional layer. Nonlinear ReLU is implemented at the output of each convolutional layer and fully connected layer. 
The kernels of the second, fourth, and fifth convolutional layers are only connected to the kernel graph of the previous layer of the same GPU. The third convolution kernel is connected to all kernel maps in the second layer. Neurons are connected to a hierarchy that is fully connected to all previous neurons in the previous layer. The response normalization layer follows the first and second convolutional layers. The largest pooling layer follows the full layer and the fifth convolutional layer. A nonlinear ReLU is implemented for the output of each convolutional layer and fully connected layer, as shown in Figure 7. The described neural network has 60,000 parameters, 650,000 neurons, 5 convolutional layers and 3 fully connected layers.

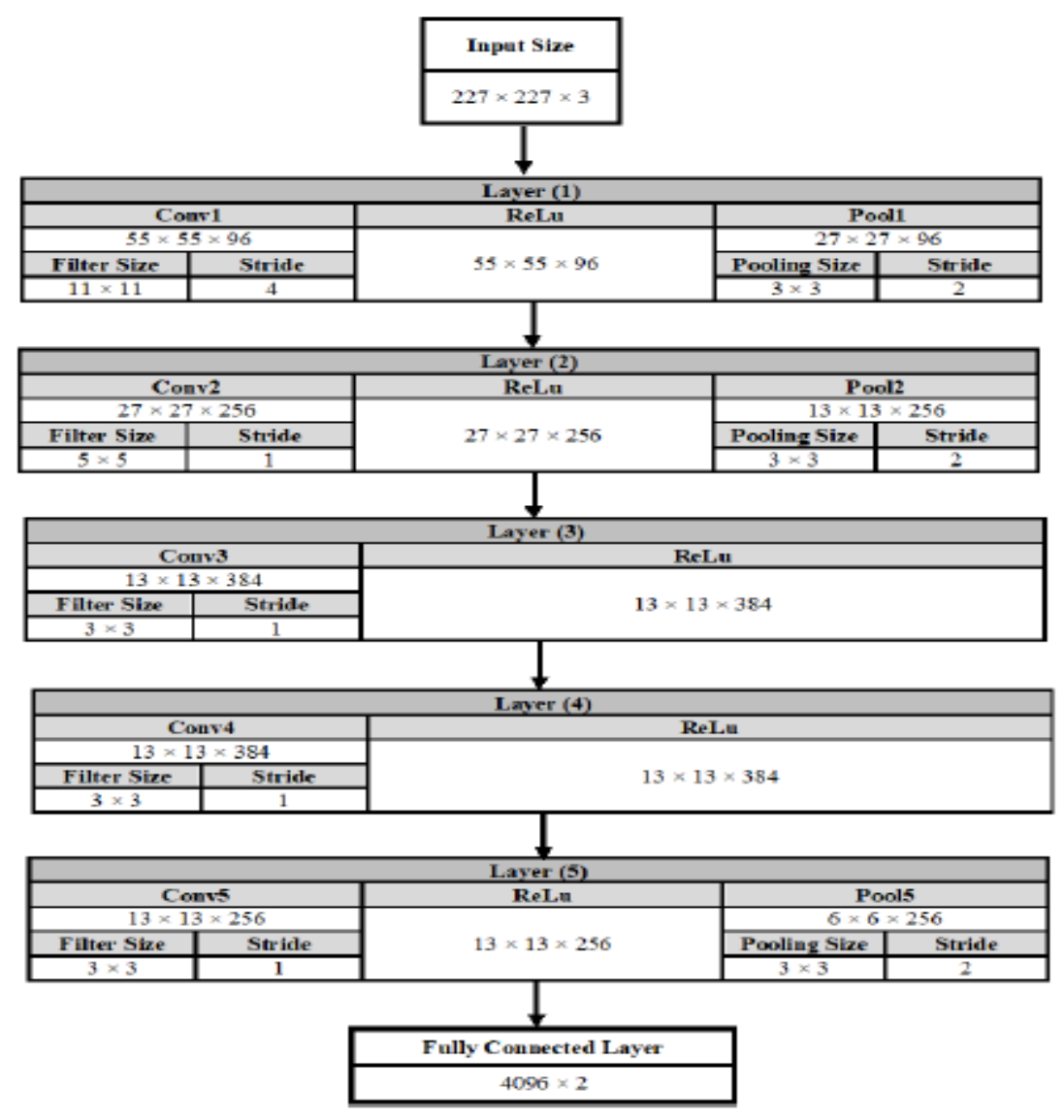

Figure 7: Double Convolutional Neural network

\section{Results and Discussions}

\subsection{Performance Metrics:}

The simulation results and performance analysis of the proposed intra-cardiac mass detection and classification with some other conventional methods are discussed in this section.

Accuracy: The percentage of correctly classified cases is often referred to as accuracy or sample accuracy. The accuracy of the system is defined as the percentage of the area classified in the ground truth specified in the equation (5), 


$$
\text { Accuracy }=\frac{T P+T N}{T P+F P+T N+F N} \ldots
$$

Sensitivity: Sensitivity is another factual estimation that is utilized to demonstrate the right pace of distinguishing proof. This is normally evaluated in terms of percentage. The mathematical formulation of sensitivity is given by equation (6),

$$
\text { Sensitivity }=\frac{T P}{F N+T P} \ldots
$$

Specificity: Specificity identifies the capacity of the decision to dismiss healthy patients without a condition. The uniqueness of this test is the number of healthy patients who know that they have cardiac issues. This is normally evaluated in terms of percentage. The mathematical formulation of specificity is given by equation (7),

$$
\text { Specificity }=\frac{T N}{T N+F P} \ldots
$$

Where, $\mathrm{TP}=$ True Positive, $\mathrm{TN}=$ True Negative, $\mathrm{FP}=$ False Positive, and FN $=$ False Negative .

\subsection{Discussions}

\begin{tabular}{|c|c|c|c|}
\hline Input Images & $\begin{array}{c}\text { Result of } \\
\text { Preprocessing }\end{array}$ & $\begin{array}{c}\text { Result of } \\
\text { Segmentation }\end{array}$ & $\begin{array}{c}\text { Type of } \\
\text { cardiac mass }\end{array}$ \\
\hline Input Image-1 & A & 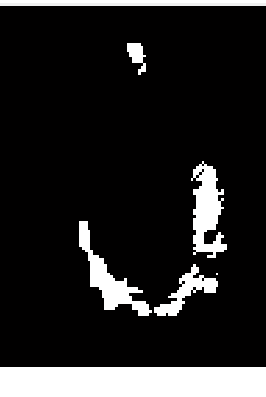 & Thrombi \\
\hline Input Image-2 & $\begin{array}{r}1 \\
2 \\
2\end{array}$ & t & Thrombi \\
\hline
\end{tabular}

The input images (image 1-6), the result of the patch based sparse representation and the LIVS are shown in Figure 7. 


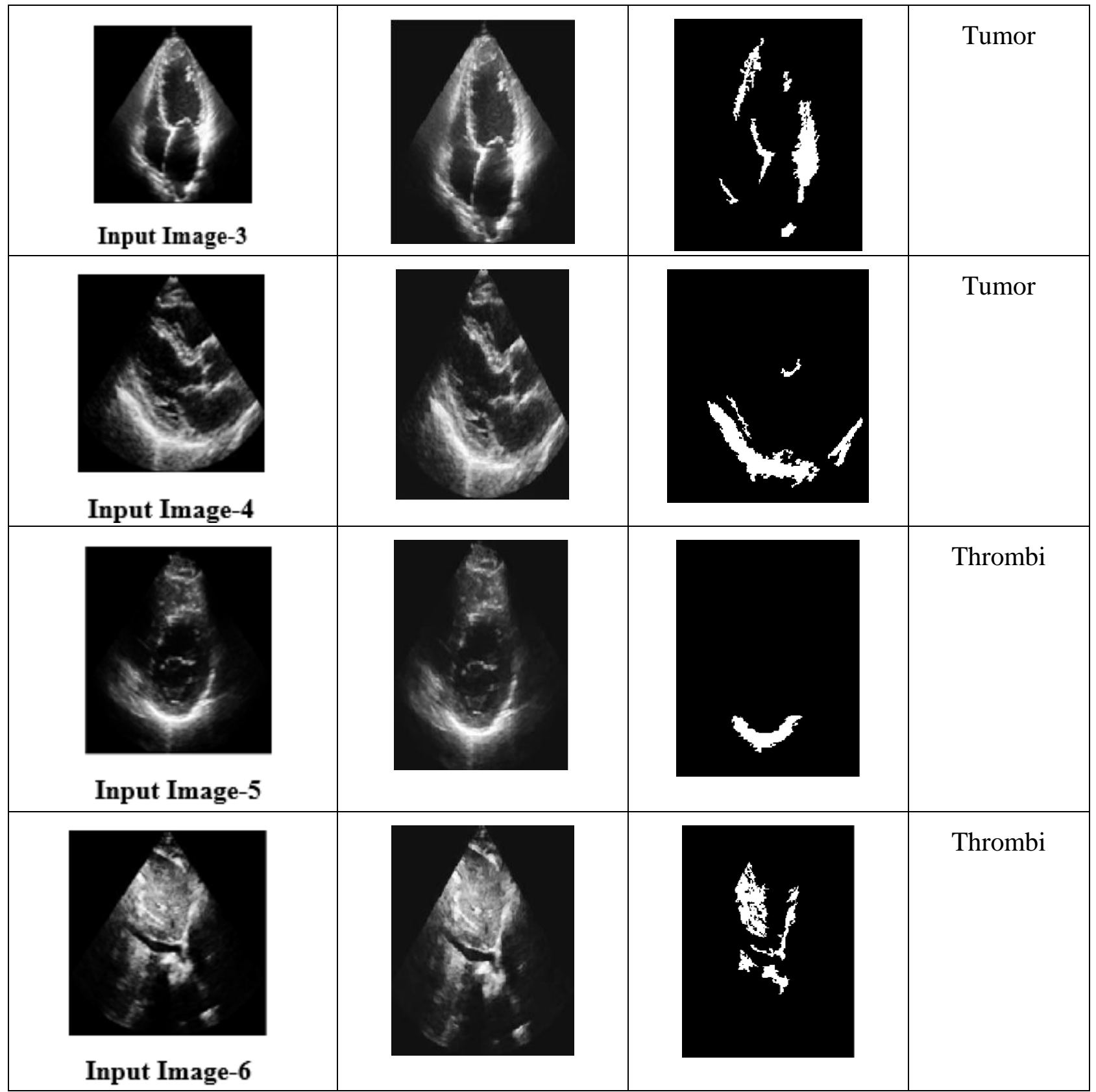

Figure 8: Preprocessing and Segmentation results of different Intra-Cardiac masses

The simulation result of the preprocessing and segmentation of different samples is shown in Figure 8. The performance analysis of preprocessing with different filters is listed in Table 2.

Table 2 depicts that introduced patch based sparse representation denoise approach attains a better response to image preprocessing. The evaluation of MSE for different echocardiogram images and are compared with various methods, and it illustrates clearly that the proposed 
method has produced a low mean squared error value and significant PSNR as compared with other filtering methods.

Table 2: Performance evaluation of filtering response

\begin{tabular}{|c|c|c|c|c|c|c|c|}
\hline Parameters & Filters & Image 1 & Image 2 & Image 3 & Image 4 & Image 5 & Image 6 \\
\hline \multirow{4}{*}{ MSE } & Gaussian & 14.394 & 13.869 & 15.698 & 14.782 & 13.698 & 15.695 \\
\cline { 2 - 8 } & Mean & 11.553 & 11.569 & 13.692 & 12.089 & 10.859 & 13.126 \\
\cline { 2 - 8 } & AVM & 7.753 & 8.693 & 9.630 & 8.963 & 7.631 & 8.425 \\
\cline { 2 - 8 } & Patch & 7.134 & 8.325 & 9.120 & 8.389 & 7.178 & 7.987 \\
\hline \multirow{4}{*}{ PSNR(in dB) } & Gaussian & 30.895 & 32.569 & 33.698 & 31.896 & 32.584 & 33.698 \\
\cline { 2 - 8 } & Mean & 33.886 & 34.875 & 37.960 & 34.871 & 35.612 & 36.742 \\
\cline { 2 - 8 } & AVM & 41.646 & 40.894 & 41.467 & 40.569 & 41.871 & 41.968 \\
\cline { 2 - 8 } & Patch & 40.567 & 40.432 & 40.675 & 40.256 & 41.287 & 41.438 \\
\hline
\end{tabular}

Table 3 discusses the global consistency error (GCE) between different methods. It measures the extent to which one segmentation can be viewed as a refinement of the other. The proposed LIVS has low GCE rate conventional K-means clustering and fuzzy C-means (FCM) clustering methods.

Table 3: Performance evaluation of Global Consistency Error

\begin{tabular}{cccccccc}
\hline Parameters & $\begin{array}{c}\text { Segmentation } \\
\text { method }\end{array}$ & Image 1 & Image 2 & Image 3 & Image 4 & Image 5 & Image 6 \\
\hline GCE & K-means clustering & 0.4634 & 0.4567 & 0.4890 & 0.4725 & 0.4604 & 0.4464 \\
& FCMclustering & 0.4378 & 0.4398 & 0.4504 & 0.4567 & 0.4306 & 0.4257 \\
& LIVS & 0.3624 & 0.3534 & 0.3467 & 0.3354 & 0.3613 & 0.3698 \\
& K-means clustering & 0.74 & 0.72 & 0.78 & 0.75 & 0.76 & 0.77 \\
DICE & FCM clustering & 0.84 & 0.85 & 0.87 & 0.86 & 0.86 & 0.89 \\
Coefficient & LIVS & 0.95 & 0.99 & 0.97 & 0.95 & 0.98 & 0.97 \\
\hline
\end{tabular}

From Table 4 and Figure 9, it is depicted that the introduced DCNN approach attains a high accuracy value while classifying the exact tumor and thrombi location from the echo-cardiogram images. The discussed system efficiency is evaluated on 6 different echo-cardiogram images. From the analysis, the DCNN attains the maximum specificity results when compared to the state of art approaches RBPNN, SVM, ANN, Sparse Representation, SVM-PSO, and KCR. The successful computation of 12 different features in the proposed method helps to recognize the intra-cardiac mass tumor with maximum accuracy $(98.89 \%)$ compared to other classifiers. The method effectively computes the output value $V_{h}=\sum_{n=1}^{N_{H i d}} w_{n h} y_{n}$ also minimizes the deviation while calculating the output value. 
Table 4: Performance analysis of accuracy with different classifier.

\begin{tabular}{cccccccc}
\hline Sample Input Images & \multicolumn{7}{c}{ Classification accuracy (\%) } \\
\cline { 2 - 8 } & ANN & SR & KCR & SVM & SVM- & RBPNN & DCNN \\
Image 1 & 85.72 & 90.32 & 91.88 & 92.02 & 93.82 & 97.35 & $\mathbf{9 8 . 6 2}$ \\
Image 2 & 84.89 & 91.47 & 92.90 & 92.93 & 94.84 & 97.39 & $\mathbf{9 8 . 3 2}$ \\
Image 3 & 85.29 & 90.58 & 91.49 & 92.40 & 94.15 & 97.58 & $\mathbf{9 8 . 9 7}$ \\
Image 4 & 85.78 & 90.98 & 92.22 & 93.93 & 94.55 & 97.85 & $\mathbf{9 9 . 1 4}$ \\
Image 5 & 85.90 & 91.03 & 91.46 & 92.95 & 93.25 & 97.69 & $\mathbf{9 8 . 8 1}$ \\
Image 6 & 84.35 & 91.48 & 92.58 & 93.85 & 94.67 & 97.61 & $\mathbf{9 9 . 2 1}$ \\
\hline
\end{tabular}

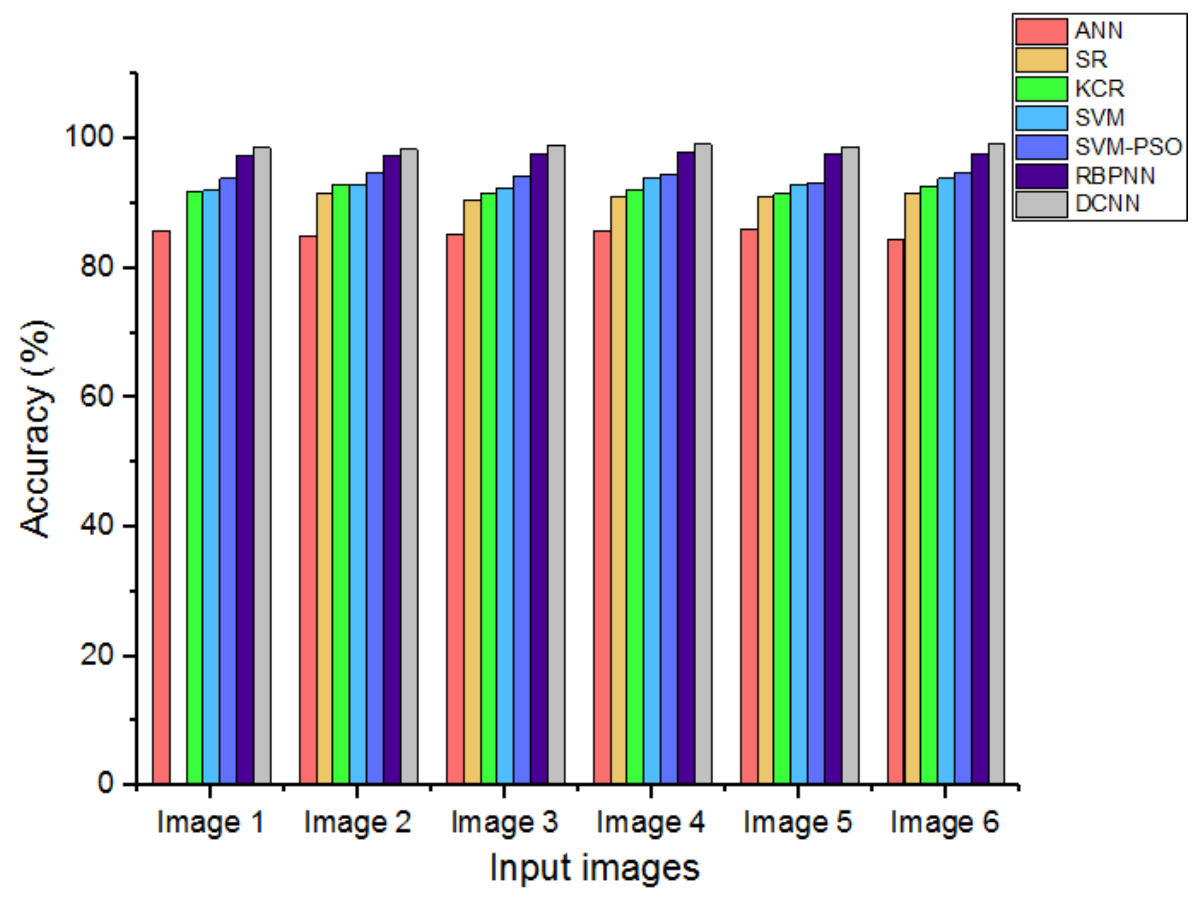

Figure 9: Result of Accuracy analysis with different classifier

From Table 5 it is depicted that the proposed DCNN approach attains the maximum sensitivity value while selecting the exact tumor location from the echo-cardiogram images. The discussed system efficiency is evaluated the six different echo-cardiogram images. From the analysis, the DCNN attains the maximum sensitivity results when compared to the state of art approaches such as RBPNN, SVM, ANN, Sparse Representation, SVM-PSO, and KCR. Based on the tabular value, sensitivity results in the graphical analysis are illustrated in Figure 10. 
Table 5: Performance analysis of sensitivity with different classifiers.

\begin{tabular}{cccccccc}
\hline Sample Input Images & \multicolumn{7}{c}{ Classification Sensitivity (\%) } \\
\cline { 2 - 7 } & ANN & SR & KCR & SVM & SVM- & RBPNN & DCNN \\
Image 1 & 92.1 & 94.56 & 95.3 & 96.29 & 96.73 & 97.29 & $\mathbf{9 8 . 4 7}$ \\
Image 2 & 93.13 & 94.89 & 95.28 & 95.78 & 96.89 & 97.389 & $\mathbf{9 8 . 6 2}$ \\
Image 3 & 92.94 & 95.13 & 95.82 & 94.9 & 97.01 & 98.18 & $\mathbf{9 8 . 9 9}$ \\
Image 4 & 92.78 & 94.47 & 94.89 & 95.28 & 96.79 & 97.38 & $\mathbf{9 7 . 8 6}$ \\
Image 5 & 93.56 & 95.05 & 94.93 & 94.38 & 96.89 & 98.98 & $\mathbf{9 9 . 1 4}$ \\
Image 6 & 93.97 & 95.25 & 95.03 & 96.309 & 97.02 & 98.23 & $\mathbf{9 9 . 6 4}$ \\
\hline
\end{tabular}

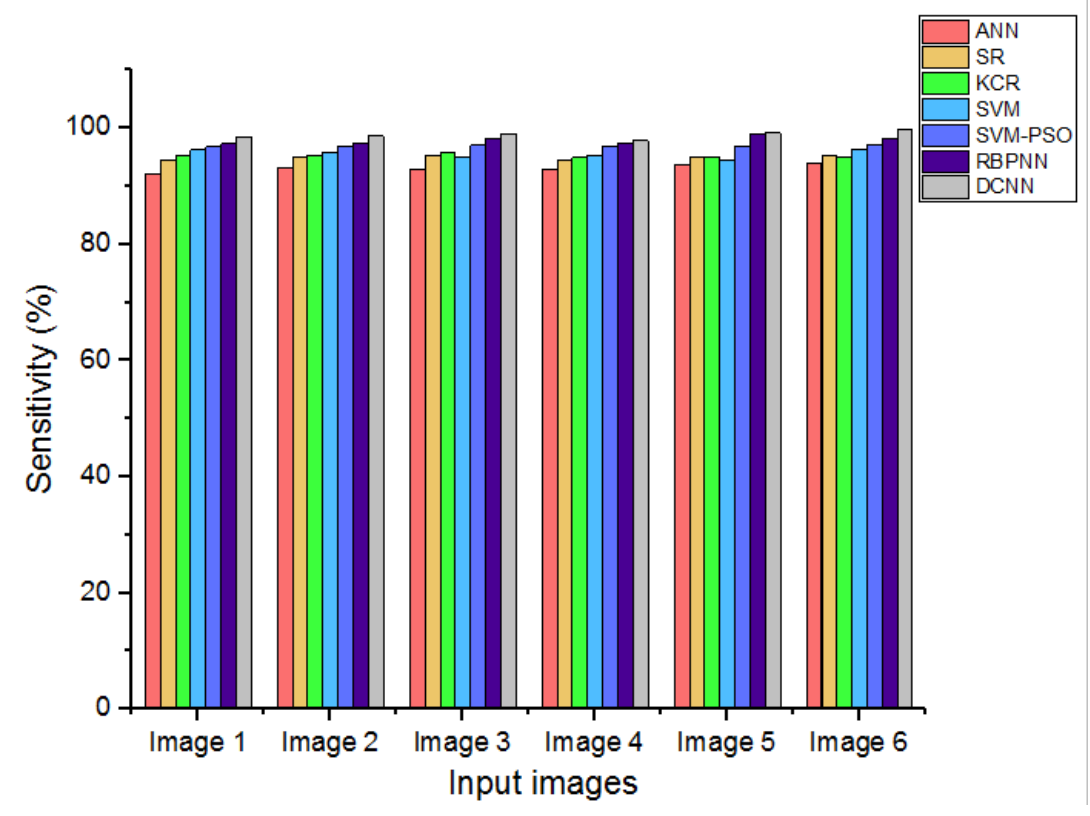

Figure 10: Result of sensitivity analysis with different classifier

From Table 6, it is depicted that DCNN approach attains the high specificity value while classifying the exact tumor location from the echo-cardiogram images. The discussed system efficiency is evaluated on 6 different echo-cardiogram images. From the analysis, the DCNN attains the maximum specificity results when the state of art approaches such as RBPNN, SVM, ANN, Sparse Representation, SVM-PSO, and KCR. Based on the tabular value, sensitivity results in the graphical analysis are illustrated in Figure 11. 
Table 6: Performance analysis of specificity

\begin{tabular}{cccccccc}
\hline Sample Input Images & \multicolumn{7}{c}{ Classification specificity (\%) } \\
\cline { 2 - 7 } & ANN & SR & KCR & SVM & SVM- & RBPNN & DCNN \\
Image 1 & 81.58 & 83.02 & 83.72 & 87.64 & 89.34 & 97.41 & $\mathbf{9 8 . 2 1}$ \\
Image 2 & 81.56 & 84.84 & 85.89 & 87.46 & 91.89 & 98.389 & $\mathbf{9 9 . 4 3}$ \\
Image 3 & 82.84 & 83.97 & 86.29 & 86.89 & 89.39 & 97.98 & $\mathbf{9 8 . 2 9}$ \\
Image 4 & 82.39 & 83.65 & 87.78 & 87.23 & 89.82 & 98.31 & $\mathbf{9 9 . 0 8}$ \\
Image 5 & 82.57 & 84.03 & 87.9 & 87.48 & 91.47 & 98.4 & $\mathbf{9 9 . 3 2}$ \\
Image 6 & 81.93 & 84.13 & 90.345 & 87.45 & 91.03 & 97.987 & $\mathbf{9 8 . 9 6}$ \\
\hline
\end{tabular}

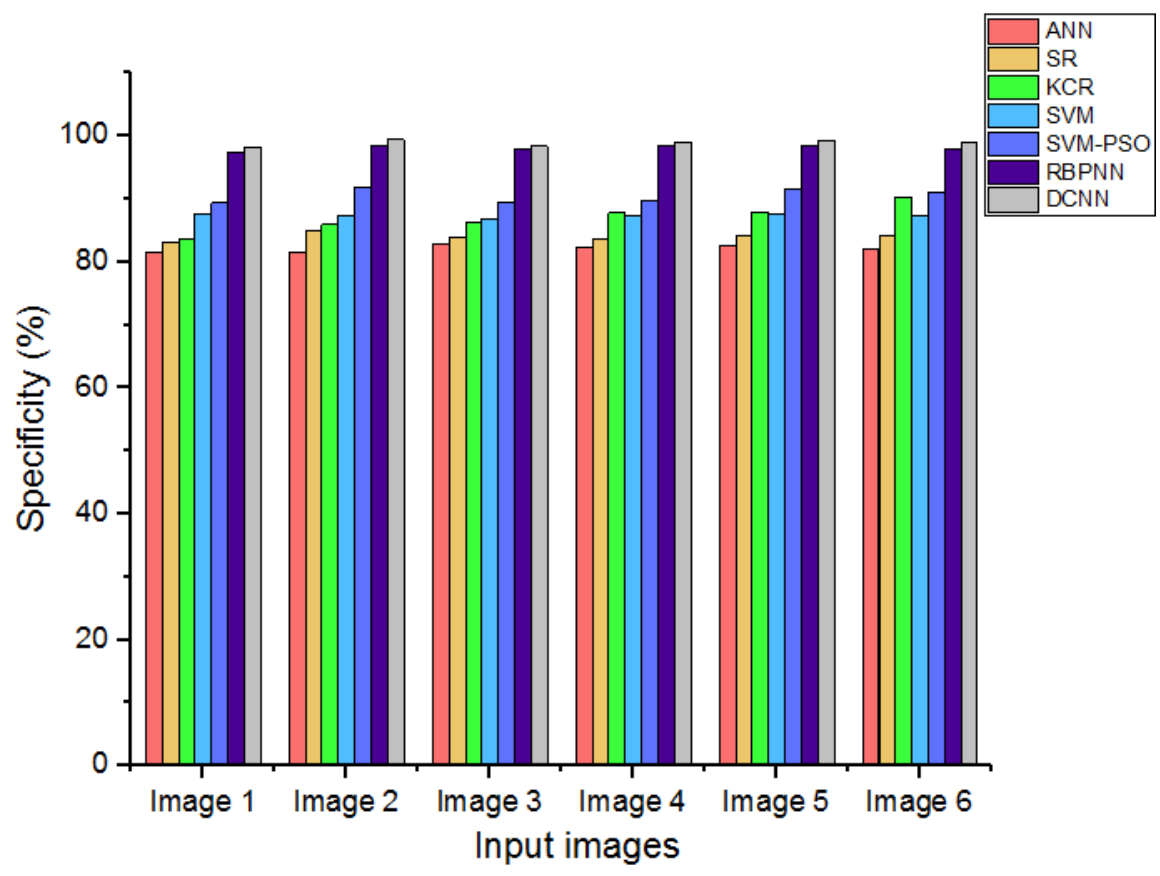

Figure 11: Result of specificity analysis with different classifier

Figure 12 and Table 7 state that the proposed DCNN method achieves the best accuracy ratio as compared with conventional methods. The Accuracy, sensitivity, and specificity suggested DCNN system are $98.98 \%, 98.89 \%$, and $99.16 \%$, respectively. 
Table 7: Comparison of the overall performance evaluation with different classifiers (Image 4)

\begin{tabular}{cccccc}
\hline $\begin{array}{c}\text { Cardiac Masses } \\
\text { Classifier }\end{array}$ & $\begin{array}{c}\text { Sensitivity } \\
(\boldsymbol{\%})\end{array}$ & $\begin{array}{c}\text { Specificity } \\
(\boldsymbol{\%})\end{array}$ & $\begin{array}{c}\text { Accuracy } \\
(\boldsymbol{\%})\end{array}$ & PPV (\%) & NPV (\%) \\
\hline SVM & 95.28 & 87.78 & 90.33 & 88.14 & 97.74 \\
ANN & 92.78 & 82.39 & 91.98 & 92.1 & 96.93 \\
SR & 94.47 & 83.65 & 92.48 & 94.74 & 96.23 \\
KCR & 94.89 & 87.23 & 93.92 & 94.98 & 95.973 \\
SVM-PSO & 96.79 & 89.82 & 94.55 & 96.29 & 95.34 \\
RBPNN & 97.38 & 98.31 & 97.85 & 98.6 & 94.5 \\
DCNN & $\mathbf{9 8 . 8 9}$ & $\mathbf{9 9 . 1 6}$ & $\mathbf{9 8 . 9 8}$ & $\mathbf{9 9 . 2 1}$ & $\mathbf{9 6 . 8 7}$ \\
\hline
\end{tabular}

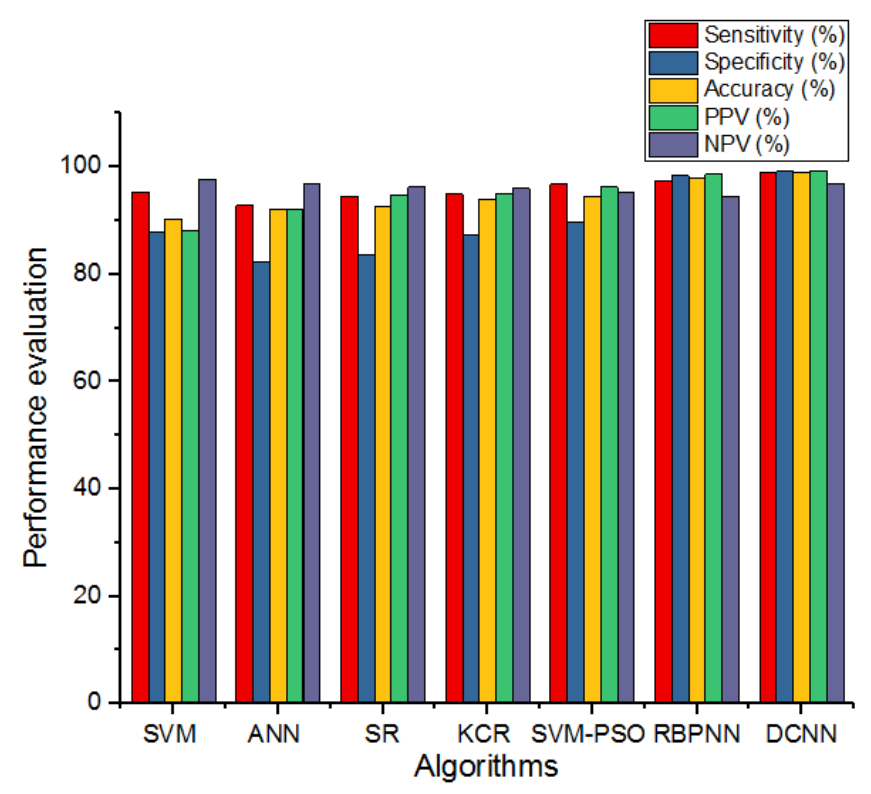

Figure 12: Overall performance analysis with different classifiers

\subsection{Run Time Analysis:}

The running time of the proposed method was calculated for each processing and it took about 435.165 seconds to analyze and identify the cardiac masses, where it took 268.78 seconds to eliminate the noise and to segment the masses, took 127.21 seconds.

\section{Conclusion}

In this work, a double convolutional neural network classifier is proposed to detect and classify intra-cardiac masses by echocardiogram images. The proposed system consists of four 
stages, such as noise removal, segmentation, feature extraction, and mass classification. Initially, the noise is diminished from echocardiogram images utilizing the patch based sparse representation. The masses were automatically segmented dependent on the LIVS strategy followed by texture features extracted utilizing the GLCM with MS-LBP method. These features were used to separate the intracardiac mass from the echocardiogram images using DCNN with a polynomial kernel for texture mixing. This research clearly states that the proposed DCNN method achieves the best accuracy ratio compared with conventional RBPNN, Sparse Representation, SVM-PSO, SVM, ANN, and KCR methods. The accuracy, sensitivity, and specificity suggested by the DCNN system are $98.98 \%, 98.89 \%$, and $99.16 \%$, More prominent productivity and basic execution make the DCNN approach helpful for cardiologists to make anticipation before medical surgery.

\section{Acknowledgements}

The authors are grateful toVinayaka Mission's KirupanandaVariyar Medical College \& Hospital, Salem, Tamilnadufor providing the Echocardiogram database of cardiac masses images.

\section{Conflict of interest:}

The authors declare that they have no conflict of interest. 


\section{REFERENCE}

[1] Jo aoPedrosa, Sandroqueir' os, Olivier Bernard, Jan Engvall, Thor Edwards ," Fast and fully automatic left ventricular Segmentation and tracking in echo-cardiographyutilizing shape-based b-spline explicit active surfaces," IEEE Trans Med Imaging. 2017 Nov;36(11):2287-2296. doi: 10.1109/TMI.2017.2734959

[2] DevisPresutti, Alberto Gomez, Graeme p. Penney and Andrew p. King," Registration of multi-view echo-cardiogramsequences utilizing a subspace error metric," IEEE Trans Biomed Eng. 2017 Feb;64(2):352-361. doi: 10.1109/TBME.2016.2550487.

[3] Douglas Wildes, Warren Lee, Bruno Haider, Scott Kogan, Krishna Kumar Sundaresan, David m. Mills, Christopher Yetter, Patrick Hart, Christopher Haun," 4d ice: a 2d array transducer with integrated ASIC in a 10 or catheter for real-time $3 \mathrm{~d}$ intra cardiacechocardiography," IEEE Trans UltrasonFerroelectrFreq Control. 2016 Dec;63(12):21592173.doi.org/10.1109/TUFFC.2016.2615602.

[4] Long $\mathrm{Yu}$, share Zhou, and bin he," temporal sparse promoting three-dimensional imaging of cardiac activation," IEEE Trans Med Imaging. 2015 Nov;34(11):2309-19. doi: 10.1109/TMI.2015.2429134..

[5] Guo Y, Wang Y, Kong D, Shu X. Automatic classification of intracardiac tumor and thrombi in echocardiography based on sparse representation. IEEE J Biomed Health Inform. 2015 Mar;19(2):601-11. doi: 10.1109/JBHI.2014.2313132. Epub 2014 Mar 21. PMID: 24691169.

[6] Hui Liu, Chung Chan, YarivGrobstein, than yu ma, Daqing Liu, "3D molecular breast imaging utilizing a high resolution dedicated cardiac SPECT camera," https://ieeexplore.ieee.org/xpl/conhome/6819718/proceeding.doi.org/10.1109/NSSMIC.2 013.6829097

[7] Yuan Xie, washing Zhang, Quichua Li, Zhuang line, any qu," Discriminative object tracking via sparse representation and online dictionary learning," IEEE Transactions on Cybernetics, vol-14, issue-4, 2014.doi.org/10.1109/TCYB.2013.2259230

[8] Dirar s. Khoury, layup rao, and DorinHanescu," Contrast echo-cardiogramfor cardiac radio-frequency ablation," IEEE Pulse. 2011 Sep-Oct;2(5):56-64. doi: 10.1109/MPUL.2011.942606..

[9] Andrij Abram yuk, Gunter wolf, Volker hints hold, Ulrike Chamberland," Comment on "developing due-it to quantify intra-tumor heterogeneity in breast tumors with differing angiogenic phenotype,” IEEE Trans Med Imaging. 2010 Apr;29(4):1088-9; 1089-92. doi: 10.1109/TMI.2009.2031780.

[10] AnirbanMukhopadhyay, Rohan Sharma Kumar," Unsupervised myocardial Segmentation for cardiac bold," IEEE Trans Med Imaging. 2017 Nov;36(11):2228-2238. doi: 10.1109/TMI.2017.2726112. 
[11] Minsongcao, Yun Liang, and Keith m. Stantz, "Developing DCE-CT to quantify intratumor heterogeneity in breast tumors with differing angiogenic phenotype" IEEE Trans Med Imaging. 2009 Jun;28(6):861-71. doi: 10.1109/TMI.2008.2012035

[12] Anita Agrawal," Estimation of cardiac parameters utilizing image processing," 2008 IEEE Region 10 and the Third international Conference on Industrial and Information Systems, Dec, 2008.10.1109/ICIINFS.2008.4798424

[13] Suyash p. Awate Edward v. R. Dibella," Model-based image reconstruction for dynamic cardiac perfusion MRI from sparse data," ConfProc IEEE Eng Med Biol Soc. 2006;1:936-41.

[14] Meng, J., Zhao, H., Liu, Y. et al. Assessment of cardiac tumors by ${ }^{18}$ F-FDG PET/CT imaging: Histological correlation and clinical outcomes. J. Nucl. Cardiol. (2020). https://doi.org/10.1007/s12350-019-02022-1

[15] Meng, J., Zhao, H., Liu, Y. et al. Assessment of cardiac tumors by ${ }^{18}$ F-FDG PET/CT imaging: Histological correlation and clinical outcomes. J. Nucl. Cardiol. (2020). https://doi.org/10.1007/s12350-019-02022-1

[16] Leiner, T., Rueckert, D., Suinesiaputra, A. et al. Machine learning in cardiovascular magnetic resonance: basic concepts and applications. J CardiovascMagnReson 21, 61 (2019). https://doi.org/10.1186/s12968-019-0575-y

[17] Burazor, I., Aviel-Ronen, S., Imazio, M. et al. Metastatic cardiac tumors: from clinical presentation through diagnosis to treatment. BMC Cancer 18,202 (2018). https://doi.org/10.1186/s12885-018-4070-x

[18] Sidharth Raj, R. S., and Dr B. Karthik. "Intra-Cardiac Tumor and Thrombi Classification in ECG based on Kernel Collaborative Representation." International Journal of Psychosocial Rehabilitation 22.4 (2018).

[19] Amano, Jun \& Nakayama, Jun \& Yoshimura, Yasuo\& Ikeda, Uichi. (2013). Erratum to: Clinical classification of cardiovascular tumors and tumor-like lesions, and its incidences. General thoracic and cardiovascular surgery. 61. 10.1007/s11748-013-0214-8.

[20] Wang JG. (2018) Primary Cardiac Tumors. In: Dumitrescu S., Ţintoiu I., Underwood M. (eds) Right Heart Pathology. Springer, Cham. https://doi.org/10.1007/978-3-319-737645_29.

[21] K.SheikAliand, Dr.M.MohamedSathik "An Innovative Approach For Detection And Classification Of Intercardiac Tumor And Thrombi" International Journal of Advanced Research in Biology Engineering Science and Technology (IJARBEST) Vol. 2, Issue 6, May 2016

[22] Venkatalakshmi.T and R.Saravanan "Intra-cardiac Tumor and Thrombi Classification in ECG Based on Kernel Collaborative Representation" International Journal of Advanced Research in Management, Architecture, Technology and Engineering - 2016

[23] Garima Singh; M. A. Ansari "Efficient detection of brain tumor from MRIs utilizing Kmeans segmentation and normalized histogram" 2016 1st India International Conference on Information Processing. 
[24] GERBER, THOMAS C., et al. "Differentiation of Intracardiac Tumors and Thrombi by Echocardiography Tissue Characterization: Comparison of an Artificial Neural Network and Human Observers." Echocardiography 17.2 (2000): 115-126.

[25] Strzelecki, Michal, et al. "Classification and segmentation of intracardiac masses in cardiac tumor echocardiograms." Computerized Medical Imaging and Graphics 30.2 (2006): 95-107.

[26] Cristina Basso, Stefania Rizzo, Marialuisa Valente, Gaetano Thiene "Prevalence and pathology of primary cardiac tumors" Cardiovascular Medicine 2012

[27] Li X, Chen Y, Liu J, et al. Cardiac magnetic resonance imaging of primary cardiac tumors. Quant Imaging Med Surg. 2020;10(1):294-313. doi:10.21037/qims.2019.11.13

[28] Meng, J., Zhao, H., Liu, Y. et al. Assessment of cardiac tumors by ${ }^{18}$ F-FDG PET/CT imaging: Histological correlation and clinical outcomes. J. Nucl. Cardiol. (2020). https://doi.org/10.1007/s12350-019-02022-1

[29] Leiner, T., Rueckert, D., Suinesiaputra, A. et al. Machine learning in cardiovascular magnetic resonance: basic concepts and applications. J CardiovascMagnReson 21, 61 (2019). https://doi.org/10.1186/s12968-019-0575-y

[30] Burazor, I., Aviel-Ronen, S., Imazio, M. et al. Metastatic cardiac tumors: from clinical presentation through diagnosis to treatment. BMC Cancer 18, 202 (2018). https://doi.org/10.1186/s12885-018-4070-x 